\title{
BLENDED LEARNING EDUCATION ON PRECISION FARMING IN \\ DEVELOPING COUNTRIES: THE EXAMPLE OF CAPE VERDE AND \\ MOZAMBIQUE
}

\author{
Prof. Dr. Marco Painho ${ }^{1}$ \\ Dr. Alexandre Baptista ${ }^{1}$ \\ Dr. Ali Atumane ${ }^{2}$ \\ Dr. Elsa Simões ${ }^{3}$ \\ ${ }^{1}$ NOVA Information Management School, Portugal \\ ${ }^{2}$ Universidade Católica de Moçambique, Mozambique \\ ${ }^{3}$ Universidade de Cabo Verde, Cape Verde
}

\begin{abstract}
The continuous development of techniques related to agriculture information management and GIS, with a close connection to sustainable development, are factors which contribute to the dissemination of precision farming.

Cape Verde and Mozambique have a strong dependence on agriculture, a lack of skilled professionals and extreme poverty rates in rural areas. There has been an increased acknowledgement of the need to develop new educational programs in developing countries focused on innovative crop and land management concepts. To overcome this issue, the NOVA Information Management School created a consortium with the Catholic University of Mozambique, the University of Cape Verde.

The consortium developed the AgIM (Agriculture Information Management and Precision Farming) master course. Working in a blended learning format, the students learn to work with GIS, remote sensing, variable rate technologies, GPS and applications for the analysis of geo-referenced data.

Currently both countries have skilled technicians with a strong interaction with local communities and farmers aiming at multiplying the benefits of precision farming, allowing to contribute to the decrease of poverty due to an improvement of the fields' management and production.
\end{abstract}

Keywords: post-graduated education, geographic information systems, blended learning, precision farming, distance learning

\section{INTRODUCTION}

In the last two decades, a growing interest has been given to a new concept of farming, known as Precision Farming. This scientific field is backed by the latest advances not only in traditional areas of agronomic science but also by the emergence and convergence of modern forms of positioning systems, computerized processing of maps and variable rate application, on what has been called computer-aided farming. In fact, the advances 
on Geographic Information Systems (GIS), Global Positioning Systems, Remote Sensing, Variable Rate Technologies or Variable Rate Applicators and Yield Monitors contributed for the concept of spatial and temporal variability to be adequately considered within the field. By exploring the potential of these technologies, the rationale of precision farming is encompassed in a new farming concept that focuses on fine-tuning of production inputs like seed, nutrient, water, pesticide, and energy and labor for smaller management units, which aim to [1]:

i) Increase production efficiency and profits

ii) Improve product quality

iii) Use of chemicals and pesticides efficiently and judiciously

iv) Preserve the energy

v) Protect soil and ground water

Precision farming has the potential to improve significantly the decision-making process in farming and to enhance agricultural profitability by supporting the economically and environmentally sustainable utilization of soil, water and nutrient resources through the integration of information- and production-based agricultural systems to optimize longterm, site-specific and whole-farm productivity while minimizing impacts on the environment.

Several factors have concurred for a situation where the adoption of precision farming is still rather limited [2]. This situation is particularly meaningful in developing countries, where the structure of agricultural production, the conditions of small farmers, the level of accessibility to well-trained, knowledge people, and the cost and availability to obtain financial support, products, and technologies is much harder.

In fact, the commonly stated reason that the benefits of precision farming are insufficient to justify the cost of its adoption seems to have been hindering the possibilities of adopting precision farming in developing countries.

However, as argued by several authors the main goal of precision farming (to optimize yield with minimum input and reduced environmental pollution to meet both food security and sustainability criteria), should be a fundamental strategy to promote sustainable agricultural development and to accelerate change in the developing countries [3], [4], [5], [6], [7].

If, on the one hand increasing land degradation in developed countries suggests that locally devised methods, on their own, are no longer effective enough to cope with rapidly changing pressures of the global market, on the other hand, considerable evidence exists that precision farming could decisively help to manage the variation of natural resources more effectively, as well as to satisfy internal demand and to meet economic and environmental standards of production [4].

Moreover, several approaches to precision farming aim to benefit from the integration of spatial information with local knowledge to develop a strategy specific to each paddock and each farm.

Indeed, the long-term experience of farmers in traditional cultures in managing specific landscape areas in a range of climatic and geographic regimes could provide valuable information for developing precision farming in developing countries [8]. The potential 
of local knowledge integration in precision farming approaches may also be used as a basis for appropriate agriculture development both within a culture, and for transferring indigenous local knowledge to other regions with similar environments [9].

In this context, specific strategies have been proposed for the adoption of precision farming in developing countries. As an example, we present the five-step strategy for the adoption of PF in India [10]:

1. Creation of multidisciplinary teams to study the overall scope of precision farming.

2. Formation of farmers' cooperatives to enable the acquisition of precision farming tools.

3. Government legislation restraining indiscriminate use of farm inputs that could cause ecological/environmental problems.

4. Carrying out pilot studies on farmers' fields to demonstrate the benefits of precision farming.

5. Creation of awareness amongst farmers of the adverse implications of applying excessive quantities of farm inputs.

In this context, there has been an increased recognition of the need to develop new agriculture educational programs in developing countries that reflects the major ongoing dynamics of change by focusing on new crop and land management concepts, in this way contributing to satisfy the growing demand of relevantly skilled professionals in agricultural and food-related jobs.

To be responsive to the specificities and changing needs of producers and agribusinesses, these programs should take into consideration a deep understanding of the specific constraints towards the implementation of precision farming in developing countries and integrate revolutionary approaches to agroecosystem management, specially by harnessing the global information and developing local precision techniques suitable for their region, operation and resources. Therefore, they should be tailored to address at the same time the expectations of the producers (giving priority to locally-developed practices and focusing on smallholder agriculture and rural livelihoods) and the broader demands of the sector.

Originally, in Mozambique and Cape Verde, there was no higher education institution offering a degree in precision farming and agriculture information management. Given the importance of agriculture to the economy of both countries and a shortage of trained specialists, NOVA Information Management School (NOVA IMS) developed a partnership with Universidade de Cabo Verde (UniCV) and Universidade Católica de Moçambique (UCM), to create a post-graduation and master course in agriculture information management and precision farming in both institutions.

\section{THE AGIM MASTER COURSE}

The AgIM - Agriculture Information Management and Precision Farming Project, was developed in collaboration between the NOVA IMS, as the applicant institution, the 
Faculdade de Agricultura (UCM), the Escola Superior de Ciências Agrárias e Ambientais (UniCV) and with the technical support of Esri Portugal and Trimble.

The Project is funded by the EDULINK II Program (financed from the European Commission) and the key objective was to implement a master course program with a curriculum based on best practices in agriculture information management and precision farming in both partner institutions, bearing in mind the new developments and specific conditions for its introduction in developing countries.

The course started in January 2014 in Mozambique (and September 2014 in Cape Verde) and covered the three major areas where improvements in precision farming can be made: Agronomic knowledge and skills, computer and Information Management Skills and Contextual and procedural skills.

The curriculum was designed to address the interdisciplinary nature of the field of agriculture and information technologies, both at the conceptual level, as well as at the level of methodologies and data analysis techniques.

The AgIM Master Programme is primarily dedicated to Cape Verde's and Mozambique's agronomy/agriculture practitioners and researchers, working in agriculture related fields and involved in decision-making and farming management tasks or in other natural resources management activities.

The course included several practical activities that rely on real demonstrations and two specific pilot demonstration projects (PDP), in this way offering students the opportunity to learn practical methods (see figure 1) for integrating precision farming into decisionmaking processes, based on the fundamental steps involved in the adoption and application of precision farming [2], namely:

1. Learning and understanding the concept of spatial data management, including the importance and value of spatial data;

2. Learning the proper use of technologies (GPS, Remote Sensing, Yield Monitoring Systems, Variable-Rate Technology) that will contribute to obtain intensive sampling of quality information at a relatively inexpensive cost;

3. Learning to use a computer and software for mapping computerized maps;

4. Understanding the relevant and manageable yield influencing factors and the extent \& stability of crop variation;

5. Conduct field work to better understand what is causing the variability (integration of local and expert knowledge);

6. Develop site-specific management plans to test the responses of managing this variability and to apply variable-rate application methods and optimizing management through strategic sampling and on-farm trials.

This methodology helped the students to develop an understanding of the various sides of the precision farming field that can be incorporated into current agricultural programs and initiatives. After completing the master programme, the students are prepared to play a significant role in the agriculture/food job market as properly skilled precision farming specialists. Consequently, they are prepared to apply problem solving skills necessary to work in a precision farming field, particularly through the implementation of the following tasks: i) collection of information about soil and field attributes, yield data or field boundaries, using field data recorders and basic geographic information systems ii) documentation and maintenance of records of precision farming information; iii) classification of agricultural fields into geo-referenced zones based on soil characteristics 
and production potentials iv) recommendation of best crop varieties and seeding rates for specific field areas, based on analysis of geospatial data.

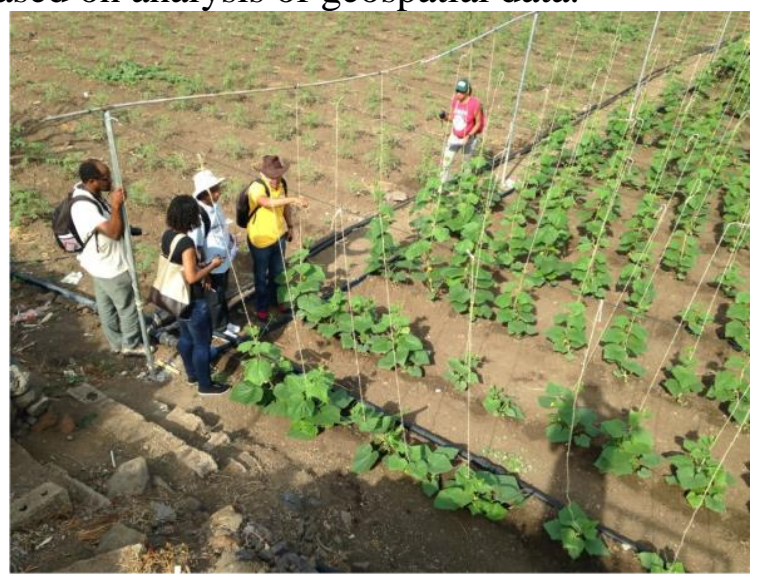

Figure 1 - AgIM students during a practical class

To foster capacity building and regional integration, the project also provided specific training courses, specifically designed to upgrade the qualifications of academic and management/administration staff in both partner institutions.

The training courses were held in both partner institutions. The following courses were prepared: problem-based learning, curriculum development theories, both targeted to the teaching staff and educational technologies for teaching and IT staff. For the academic and management staff, it was developed a course on international project management. Two technical courses were organized, with the participation of students and local agriculture technicians: the current state of the art of precision farming, where the attendees received training in the latest techniques in precision farming and UAV AgIM. This course was dedicated to drones and its using in precision farming.

Through the implementation of two pilot demonstration projects, the consortium is currently developing a repository of good practices that will support the exchange of experiences, promoting the development of meaningful skills targeting to address the specific needs of small farmers and local communities.

The pilot demonstration projects promoted a close collaboration between small farmers, rural communities, researchers, teachers and students, as well as the creation of an information base and groundwork that will further support the implementation of precision farming concepts, methods and techniques in the adaptation and actualization of existing agricultural curricula.

To guarantee the sustainability of the course after the implementation period, the classes were originally taught by NOVA IMS teachers and 3 local teachers ( 2 from Mozambique and another from Cape Verde). To guarantee the sustainability of the project, after the course's first edition, the best students were invited to assist the teachers in class, creating a new teaching staff for the remaining editions.

\section{BLENDED LEARNING MODEL IN AGIM}

Blended learning can be defined as an integrated combination of traditional learning (face-to-face classes) with web based on-line approaches. The delivery of the online part 
of a blended learning course is usually through learning technologies, typically involving a Virtual Learning Environment (VLE), such as Blackboard or Moodle [11]. The communication between the learner and e-tutor may take place through a chat system or forum in the VLE platform or by email. One of the advantages of adopting blended learning if the fact that the platform is always available anytime and anywhere. The only schedule that the students need to follow are the presential classes and the session of discussion of the projects developed during the curricular units [11].

In the AgIM course, each curricular unit has a duration of 5 weeks. The methodology for delivering the classes is as follows: in the first week of each curricular unit, the teacher uploads the learning materials such as exercises, presentations, reports, videos, etc. in the Moodle platform (the implemented VLE for the course). During that week, the students study and analyze the learning materials for preparing for the second week which is dedicated to the presential classes. There, the students have intensive face-to-face classes and take an exam at the end of the week. The last three weeks are dedicated to the development of a final project, to be presented at the end of the semester.

\section{A COURSE STRUCTURED BY GEOSPATIAL TOOLS}

Each edition has a duration of 3 semesters, the first two are dedicated to the curricular module of the course, where all the curricular units are of mandatory nature (four curricular units per semester). The last one is focused on the development of the dissertation. The attribution of the master's degree requires, apart from approval in the curricular component of the $1^{\text {st }}$ year, the preparation, discussion and approval of an original scientific thesis or project work.

The first semester is dedicated to introductory curricular units on the precision farming and GIS themes: GIS and Precision Farming, Research Methods, Data Acquisition and Remote Sensing and Spatial Analysis.

In GIS and Precision Farming, where the students learn the bases of GIS and its role in the development of precision farming. In Data Acquisition, the students gather information (using GPS devices) in local agriculture fields, that are used for the pilot demonstration projects. Taught by a local teacher, this curricular unit is vital, due to field work experience, the importance of the collected data that is used by the students as case studies in the PDP's, in some of the student's dissertations and are also used in the following curricular units. The Remote Sensing and Spatial Analysis unit, is focused on the principles of remote sensing, identify the main sources of geographic data for creation, edition and modelling.

The second semester focuses on a set of four courses with a higher level of specialization and complexity: Quantitative Methods, Precision Farming, Agriculture Information Management and Implementation of Precision Farming in the Community.

In Quantitative Methods, the students learn to explore and interpolate geographic data. The Precision Farming is based on the use of spatial information in the design of intelligent soil sampling and learn the importance of geoelectric sensors in the analysis of spatial variability of soil quality. In Implementation of Precision Farming in the 
Community, the students learn the concept of Public Participation GIS and how to create it.

After analyzing the 8 curricular units, we can conclude that the course is based in six main learning areas:

1. Sustainable Agriculture

2. Precision Farming - Conceptual Foundations

3. Precision Farming - Analytical Methods

4. Agriculture and Geospatial Technologies

5. Agricultural Information Management

6. Geographic Information Systems.

The course is extremely focused on the use of geospatial tools on precision farming. From the eight curricular units of the course, only two, Research Methods (research and organizational skills) and Agriculture Information Management (database management), do not have in the curriculum the use of geospatial tools.

At the end of each semester, the students present and discuss the final projects (one per curricular unit). The discussion sessions are open to the academic staff and the community in general.

\section{CONCLUSION}

The main impact of the project is the qualification of a group of skilled technicians that successfully met the fundamental steps involved in the adoption and application of precision farming, having a better understanding of small-scale producers' needs and in possession of knowledge and skills to improve significantly the decision-making process and to enhance agricultural profitability that can play a decisive role both in Cape Verde and Mozambique, especially in reducing rural poverty, improve food security and sustain good practices on natural resources management.

The capacity of both partner higher education institutions was improved and the human capital strengthened, through knowledge transfer in the technical, didactical and scientific domains, allowing for a gradual transition, based on quality criteria and adequate resource and technology use, necessary for the sustainability of the Action.

To ensure the sustainability of the course at class level, during the second edition of the AgIM Master Programme, the teaching staff was reinforced with the direct involvement the best students selected from the first edition. This methodology proved to be successful, especially in Mozambique, that already organized two editions independently from the project.

The project also implemented a set of procedures and methods (communication and dissemination plans, guidelines for project management, accreditation dossiers, PDP plan, procedures for evaluation and quality assurance standards, etc.), that are 
contributing decisively to improve the management and financial administration capacity of both partner institutions.

The partners also benefited from the contributions of the associate partners, who provided the software and the education resources necessary for the implementation of the AgIM master program.

\section{ACKNOWLEDGEMENTS}

This work was supported by the EDULINK II, funded by the European Commission and implemented by the ACP (African, Caribbean and Pacific Group of States) Secretariat under Grant FED/2013/320-079.

\section{REFERENCES}

[1] K. Adhikari, F. Carre, G. Toth, and L. Montanarella, "Site Specific Land Management: General Concepts and Applications," European Commission: Joint Research Centre - Institute for Environment and Sustainability, Luxembourg, 2009.

[2] N. R. Kitchen, C. J. Snyder, D. W. Franzen, and W. J. Wiebold, "Educational Needs of Precision Agriculture," Precis. Agric., vol. 3, no. 3, pp. 341-351, 2002.

[3] W. Maohua, "Possible adoption of precision agriculture for developing countries at the threshold of the new millennium," Comput. Electron. Agric., vol. 30, no. 13, pp. 45-50, Feb. 2001.

[4] A. Dobermann, S. Blackmore, S. E. Cook, and V. I. Adamchuk, "Precision Farming: Challenges and Future Directions," in 4th International Crop Science Congress, 2004, pp. 1-19.

[5] M. L. Rilwani and I. Ikhuoria, "Review Article Precision Farming with Geoinformatics: A New Paradigm for Agricultural Production in a Developing Country," Trans. GIS, vol. 10, no. 2, pp. 177-197, 2006.

[6] P. Mondal and M. Basu, "Adoption of precision agriculture technologies in India and in some developing countries: Scope, present status and strategies," Prog. Nat. Sci., vol. 19, no. 6, pp. 659-666, Jun. 2009.

[7] T. A. Lumpkin, "Help for developing countries in a world of rising grain prices," XXVIII Int. Hortic. Congr. Sci. Hortic. People, vol. 916, no. 916, pp. 31-46, 2010.

[8] J. A. Sandor, A. M. G. A. WinklerPrins, N. Barrera-Bassols, and J. A. Zinck, "The heritage of soil knowledge among the world's cultures," in Footprints in the Soil: People and Ideas in Soil History, B. Warkentin, Ed. Amsterdam: Elsevier, 2006, pp. 43-84.

[9] M. A. Altieri, F. R. Funes-Monzote, and P. Petersen, "Agroecologically efficient agricultural systems for smallholder farmers: contributions to food sovereignty," Agron. Sustain. Dev., vol. 32, no. 1, pp. 1-13, 2012.

[10] A. Mishra, K. Sundaramoorthi, R. Chidambara, and D. Balaji, "Operationalization of precision farming in India," Proc. Map India Conf., p. 8, 2003.

[11] P. Sharma, "Blended learning," English Lang. Teach. J., vol. 4, no. 64, 2010. 